\title{
TRANSFORMATION KINETICS FOR NUCLEATION ON RANDOM PLANES AND LINES
}

\author{
ELENA Villa ${ }^{1}$ AND PAUlo R Rios ${ }^{2}$ \\ ${ }^{1}$ Dept. of Mathematics, University of Milan, Via Saldini 50, 20133, Milano, Italy; ${ }^{2}$ Universidade Federal \\ Fluminense,Escola de Engenharia Industrial Metalúrgica de Volta Redonda, Av. dos Trabalhadores 420, \\ 27255-125, Volta Redonda, RJ, Brasil \\ e-mail: elena.villa@unimi.it, prios@metal.eeimvr.uff.br \\ (Accepted October 2, 2011)
}

\begin{abstract}
Birth and growth processes are known in materials science as nucleation and growth processes. In crystalline materials nucleation almost always takes place in an internal crystalline defect. These defects are classified according to their dimensionality: point, line or planar defects. Therefore, investigating nucleation on sets of dimensionality lower than the set in which the transformation takes place is of paramount importance. Cahn (1956) in a classical work derived expressions for transformation kinetics when nucleation took place on random planes and on random straight lines. He used these expressions to describe nucleation in polycrystalline materials. He considered that nucleation on grain faces could be treated as nucleation on random planes and, likewise, nucleation on grain edges could be treated as nucleation on random lines. The present work revisits and generalizes Cahn's treatment of nucleation on planes and lines. First a general expression for the case of nucleation on lower dimensional sets is obtained. After that general expressions for nucleation on random planes and random lines are given. This paper provides the mathematical basis for the development of more specific expressions to be used in practical applications. Although this work has been done bearing applications to materials science in mind the results obtained here may be applied to birth and growth processes in any field of science.
\end{abstract}

Keywords: birth-and-growth process, formal kinetics, phase transformations, point process, Poisson process, random set, recrystallization.

\section{INTRODUCTION}

Formal kinetics is frequently employed to analyze a variety of heterogenous transformations in condensed phases. This methodology has its origin in the early work by Kolmogorov (1937), Johnson and Mehl (1939) and Avrami (1939; 1940; 1941) and is often called KJMA theory. Heterogeneous transformations may be defined as those transformations in which there is a moving boundary between the transformed and untransformed region. This formalism envisages that the heterogeneous transformations may be decomposed in two stages. The first stage, the nucleation, is that in which the transformed region originates. On the other hand, the second stage, the growth stage, is that in which the transformed region grows consuming the parent matrix. These processes are usually called nucleation and growth processes in materials science or also birth and growth processes in mathematics. The application of this formalism is by no means restricted to materials science. Tomellini and Fanfoni (2008) pointed out that it is applied to a variety of situations, such as, the phase separations in multicomponent alloys (Starink, 2004), the film growth on solid substrates
(Fanfoni and Tomellini, 2005), the kinetics of Ising lattice-gas model (Ramos et al., 1999), and the DNA replication (Jun and Bechhoefer, 2005). To these we may add a recent extensive work by Aquilano et al. (2009) on crystallization processes.

In materials science, the applicability of KJMA formalism may be extended to transformations in which nucleation and growth are treated as purely "operational" concepts. Therefore, examples of formal kinetics modeling can be found associated with a variety of transformations, such as, austenite to perlite transformation (Johnson and Mehl, 1939), recrystallization (Vandermeer and Jensen, 2001), abnormal grain growth in $\mathrm{BaTiO}_{3}$ (Rios et al., 1998; Kondo et al., 1998), martensite "spread" (Rios and Guimaraes, 2007; 2008) and polymer crystallization (Burger et al., 2002a;b).

Specifically, nucleation in crystalline materials almost always takes place in an internal crystalline "defect". These defects are classified in three kinds according to their dimensionality: point, line or planar defects. A good example is a polycrystalline material. In this case the grain boundaries are planar defects, the common edge belonging to three grains is a line defect and the vertex(or "corner") that is 
common to four grains is a point defect. Classical nucleation theory demonstrates the importance of these internal defects as nucleation sites. According to the classical nucleation theory, the free energy barrier for nucleation strongly depends on which boundary site the nucleus is located. The free energy barrier is smallest for vertices and progressively larger for nucleation on edges and faces (Cahn, 1956). Even in transformations in which classical nucleation theory is thought not to be applicable, for example, recrystallization, grain boundary sites are often the places at which the transformation originates. Other examples of nucleation sites are lattice vacancies, dislocation lines and the particle/matrix interface in the case that the polycrystal contains a particle dispersion. In addition to internal defects in small specimens like powders, thin wires or thin films nucleation on the external surface give a significant contribution to the transformation (Villa and Rios, 2010). From these examples, it is clear that nucleation on sets of dimensionality lower than the set in which the transformation takes place is of paramount importance.

In its original form KJMA theory considered that nucleation sites were uniform randomly located in space. In other words, that the internal defects were point defects and that they were located in space according to a homogeneous Poisson point process. Expressions for two fundamental nucleation modes were obtained. The first fundamental nucleation process takes places when the nuclei are "potent" so that nucleation takes place very fast and all possible nucleation sites are exhausted very early in the reaction. This case is called "site-saturation". Mathematically speaking one may suppose that all transformed regions originate at the start of the reaction taken to be the time origin, $t=0$. The second fundamental nucleation mode considered that the internal point defects were not equally "potent". As a consequence there was a different "incubation" time for each site to become a new transformed region. Therefore nucleation took place over time with what they called a "constant nucleation rate". In mathematical terms they supposed that nucleation was a point process in $\mathbb{R}_{+} \times \mathbb{R}^{d}$. It is clear that KJMA considered only one possibility of nuclei distribution in space whereas in real materials, as discussed above, there are many possibilities.

Many metallic and ceramic engineering materials are polycrystalline. As mentioned above, nucleation of a transformed region in polycrystals does often take place at grain boundaries, edges and vertices. Grain boundaries are not exactly flat neither grain edges are exactly straight lines. Nonetheless the accurate analytical description of these boundaries and lines in a real polycrystal would be unfeasible. Clearly, it is necessary to represent grain boundaries and edges by simple curves or surfaces. Cahn (1956) in a classical work proposed that grain faces may be approximated by random planes and grain edges by random straight lines provided that there is an equivalence between the area per unit of volume of the real grain boundaries and the area per unit of volume of the random planes. Cahn's paper has been extensively cited not only in connection with metallic materials recrystallization and phase transformations, but also in connection with non-metallic materials as well, such as, glass, cement and even geological formations.

Cahn's method has two main characteristics. The first is that it captures the "randomness" in the location of boundary sites. This randomness is a consequence of the randomness in the distribution of grain sizes and of the spatial location of the grains. The second characteristic is that it also captures the dimensionality of the nucleation site. Thus distinct formal kinetics expressions are obtained for nucleation in each boundary site. Dimensionality of the operative nucleation site is clearly a key issue for transformations taking place in polycrystalline materials. These characteristics are probably what has prompted metallurgists to continually apply Cahn's model to many transformation problems.

One complication that may arise in practice is that metallic polycrystalline materials may be and indeed they are often deformed. The deformation may change the spatial arrangement of the grain boundaries. For example, after severe rolling the grains are "flattened" and are often called "pancake" grains. Clearly these grains have most of their grain boundaries nearly parallel to the rolling plane. In this case the grain boundaries might be approximated by parallel planes. Therefore depending on how the deformation is conducted the spatial arrangement of planes or lines may assume some specific characteristics. So it is of practical interest to study how different spatial arrangements of planes or lines may affect the transformation originating from nuclei on these planes or lines. This is the main goal of the present paper.

In previous work (Rios and Villa, 2009; Villa and Rios, 2009; 2010), the present authors resorted to the causal cone (Jackson, 1974; Cahn, 1996) concept and to recent developments in stochastic geometry (Capasso and Villa, 2007a;b) to obtain analytical expressions for transformations in which nuclei were located in space according to an inhomogeneous Poisson point processes, in spherical clusters and in the bulk and on the surface of small specimens (Villa and Rios, 2010). In this work we continue to examine situations of engineering and 
scientific interest exerting the same mathematical tools adopted in our previous work. In the present work we revisit and generalize the problem of nucleation on planes and lines treated by Cahn (1956) in the aforementioned paper.

This paper is organized as follows. First a short mathematical background is given. Then a general expression for the case of nucleation on lower dimensional sets is obtained. After that the mathematical derivations of general expressions for nucleation on random planes are given. The next section is similar to the previous one excepting that it treats lines instead of planes. Additional mathematical background may be found in our previous papers and references therein (Rios and Villa, 2009; Villa and Rios, 2009; 2010).

\section{MATHEMATICAL BACKGROUND AND BASIC NOTATION}

Detailed mathematical background may be found in previous work by the authors (Rios and Villa, 2009; Capasso and Villa, 2007a;b). Here only some essential definitions and some useful relationships will be presented to make this paper more self contained and easier to read. For a brief description of homogeneous and inhomogeneous Poisson point process, the reader is referred to Rios and Villa (2009) or for a more detailed presentation to specific texts on stochastic geometry (Stoyan et al., 1995).

\section{BIRTH-AND-GROWTH PROCESSES AND MEAN RELATED DENSITIES}

A birth-and-growth (stochastic) process is a dynamic germ-grain model (Stoyan et al., 1995), used to model situations in which nuclei (germs) are born in time and are located in space randomly, and each nucleus generates a grain evolving in time according with a given growth law. Since, in general, the nucleation is random in time and space, then the transformed region at any time $t>0$ will be a random set (Stoyan et al., 1995) in $\mathbb{R}^{d}$, that is a measurable map from a probability space to the space of closed subsets in $\mathbb{R}^{d}$. Denote by $T_{j}$ the $\mathbb{R}_{+}$-valued random variable representing the time of birth of the $j$-th nucleus, and by $X_{j}$ the $\mathbb{R}^{d}$-valued random variable representing the spatial location of the nucleus born at time $T_{j}$. Let $\Theta_{T_{j}}^{t}\left(X_{j}\right)$ be the grain obtained as the evolution up to time $t \geq T_{j}$ of the nucleus born at time $T_{j}$ in $X_{j}$; then, the transformed region $\Theta^{t}$ at time $t$ is

$$
\Theta^{t}=\bigcup_{T_{j} \leq t} \Theta_{T_{j}}^{t}\left(X_{j}\right), \quad t \in \mathbb{R}_{+} .
$$

The family $\left\{\Theta^{t}\right\}_{t}$ is called birth-and-growth process. Birth-and-growth and nucleation and growth will be used as synonyms in this paper.

Since $\Theta^{t}$ is a random set, it gives rise to a random measure $v^{d}\left(\Theta^{t} \cap \cdot\right)$ in $\mathbb{R}^{d}$ for all $t>0$, having denoted by $v^{d}$ the $d$-dimensional Lebesgue measure in $\mathbb{R}^{d}$. In particular, it is of interest to consider the expected volume measure $\mathbb{E}\left[v^{d}\left(\Theta^{t} \cap \cdot\right)\right]$ and its density (i.e., its Radon-Nikodym derivative), called mean volume density of $\Theta^{t}$ and denoted by $V_{V}$, provided it exists:

$$
\mathbb{E}\left[v^{d}\left(\Theta^{t} \cap A\right)\right]=\int_{A} V_{V}(t, x) \mathrm{d} x \quad \forall A \in \mathscr{B}_{\mathbb{R}^{d}},
$$

where $\mathscr{B}_{\mathbb{R}^{d}}$ is the Borel $\sigma$-algebra of $\mathbb{R}^{d}$.

Whenever $A$ is the region of the physical sample under observation, the ratio

$$
\mathbf{V}_{\mathbf{V}}(t, A):=\frac{\int_{A} V_{V}(t, x) \mathrm{d} x}{v^{d}(A)}
$$

is also called volume fraction. Let us notice that whenever $V_{V}$ is independent of $x$ (e.g., under assumptions of homogeneous nucleation and growth), then $\mathbf{V}_{\mathbf{V}}$ is independent of $A$ and $\mathbf{V}_{\mathbf{V}}(t)=V_{V}(t)$. (see also Rios and Villa, 2009, Stoyan et al., 1995, p. 342). We also mention that other quantities of interest in real applications are the so-called mean extended volume density at time $t$, denoted by $V_{E}(t, \cdot)$, defined as the density of the mean extended volume measure at time $t, \mathbb{E}\left[\mu_{\Theta^{t}}^{\mathrm{ex}}\right](\cdot):=\mathbb{E}\left[\sum_{j: T_{j} \leq t} v^{d}\left(\Theta_{T_{j}}^{t}\left(X_{j}\right) \cap \cdot\right)\right]$ on $\mathbb{R}^{d}$, that is

$$
\mathbb{E}\left[\mu_{\Theta^{\mathrm{ex}}}^{\mathrm{ex}}\right](A)=\int_{A} V_{\mathrm{ex}}(t, x) \mathrm{d} x, \quad \forall A \in \mathscr{B}_{\mathbb{R}^{d}},
$$

and the mean surface density $S_{V}(t, \cdot)$ and the mean extended surface density $S_{E}(t, \cdot)$ at time $t$, defined as the density of the mean surface measure at time $t, \mathbb{E}\left[\mu_{\partial \Theta}\right](\cdot):=\mathbb{E}\left[\mathscr{H}^{d-1}(\partial \Theta \cap \cdot)\right]$ and of the mean extended surface measure at time $t, \mathbb{E}\left[\mu_{\partial^{t}}^{e x}\right](\cdot):=$ $\mathbb{E}\left[\sum_{j: T_{j} \leq t} \mathscr{H}^{d-1}\left(\partial \Theta_{T_{j}}^{t}\left(X_{j}\right) \cap \cdot\right)\right]$, respectively, where $\mathscr{H}^{d-1}$ is the $(d-1)$-dimensional Hausdorff measure. In other words, the mean extended volume and surface measures represent the mean of the sum of the volume measures and of the surface measures of the grains which are born and grown until time $t$, supposed free to grow, ignoring overlapping (see also Rios and Villa, 2009; Villa, 2008).

It is clear that to find out formulas for the mean volume density $V_{V}$ (and so for $\mathbf{V}_{\mathbf{V}}$ and the other quantities we mentioned above, as a consequence) is of particular interest in real applications.

Of course, different kinds of nucleation and growth models gives rise to different kinds of processes $\left\{\Theta^{t}\right\}_{t}$. 
Aim of this paper is the study of the mean volume density of birth-and-growth processes with different types of nucleations on planes and lines, and so, for sake of simplicity, we shall assume throughout the paper that the velocity of growth of each grain is constant and homogeneous in space, so that any grain $\Theta_{T_{j}}^{t}\left(X_{j}\right)$ born in $X_{j}$ at time $T_{j}$ and grown up to time $t$ is given by the ball $B_{G\left(t-T_{j}\right)}\left(X_{j}\right)$ centered in $X_{j}$ with radius $G\left(t-T_{j}\right)$. Such a simplification does not limit the applications of our results to real situation; moreover the interested reader in the more general case of non-constant velocity could generalize our results by applying the same approach presented here. We also refer to Burger et al. (2002a); Capasso and Villa (2007a); Villa (2008) for models of birth-and-growth processes whose grains have space and time dependent growth rate.

\section{MODELLING THE NUCLEATION PROCESS}

As mentioned in the Introduction, we shall consider the case in which all the nucleation takes place at $t=0$ (namely, site-saturated case), and the case in which the nucleation takes place in time (namely, time-dependent case).

Site-saturated nucleation processes and spacetime dependent nucleation processes can be modeled by point processes and marked point processes, respectively. We give here some basic concepts and definitions useful for the sequel (see also, for instance, Rios and Villa, 2009, Sec. 2.3) We remind that a point process in $\mathbb{R}^{d}$ is an almost surely locally finite sequence of points $\left\{X_{i}\right\}_{i}$ randomly located in $\mathbb{R}^{d}$, according with a given probability law. It can be described by the counting process, say $N$, associated to the sequence $\left\{X_{i}\right\}$ defined as

$$
N(A):=\text { number of the } X_{i} \text { 's, which belong to } A,
$$

for any $A \in \mathscr{B}_{\mathbb{R}^{d}}$.

A marked point process in $\mathbb{R}_{+} \times \mathbb{R}^{d}$, is a sequence $N:=\left\{\left(T_{i}, X_{i}\right)\right\}_{i}$ of points in $\mathbb{R}_{+} \times \mathbb{R}^{d}$ such that the sequence $\left\{T_{i}\right\}_{i}$ is a point process in $\mathbb{R}_{+}$, while each $X_{i} \in \mathbb{R}^{d}$ is said to be the mark associated to the point $T_{i}$. A space-time nucleation process can be modeled by a marked point process, identifying $T_{i}$ as the time of birth of the $i$-th nucleus, and $X_{i}$ as its spatial location in $\mathbb{R}^{d}$. Analogously to the site-saturation case, a counting process $N$ on $\mathbb{R}_{+} \times \mathbb{R}^{d}$ can be defined as

$$
\begin{aligned}
N\left([s, t] \times A^{\prime}\right):= & \text { number of nuclei, which are } \\
& \text { born in } A^{\prime} \text { during the time } \\
& \text { interval }[s, t] .
\end{aligned}
$$

The measure $\Lambda$ on $\mathbb{R}^{d}$ and on $\mathbb{R}_{+} \times \mathbb{R}^{d}$, respectively, defined as $\Lambda(A):=\mathbb{E}[N(A)]$ for all $A \in \mathscr{B}_{\mathbb{R}^{d}}$ and $A \in$ $\mathscr{B}_{\mathbb{R}_{+}} \times \mathscr{B}_{\mathbb{R}^{d}}$, respectively, is called intensity measure of $N$; in other words, $\Lambda(A)$ represents the mean number of nuclei born in $A \subset \mathbb{R}^{d}$ of a site-saturated process, and the mean number of nuclei born in $A^{\prime}$ during a time interval $[s, t]$, where $A=[s, t] \times A^{\prime} \subset \mathbb{R}_{+} \times \mathbb{R}^{d}$, of a time-dependent nucleation. We intentionally use the same notation $N$ for the site-saturated nucleation process and for the time-dependent one because the site saturated process may be seen as a particular case of the time-dependent one by assuming $T_{j} \equiv 0$ for any $j$. In the sequel it will be clear from the context which process we are referring to (note also that $\Lambda$ is a measure on $\mathbb{R}^{d}$ in the site saturated case, and on $\mathbb{R}_{+} \times \mathbb{R}^{d}$ in the time-dependent case).

In this paper we shall assume Poissonian nucleation, that is $N$ will be a Poisson process (in $\mathbb{R}^{d}$ in the site-saturated case, and in $\mathbb{R}_{+} \times \mathbb{R}^{d}$ in the timedependent case). We point out that the resulting birthand-growth process is then a particular case of the well-known general Boolean model (Matheron, 1975); namely, due to the assumption of constant velocity, a time dependent Boolean model of spheres.

\section{CAUSAL CONE AND MEAN VOLUME DENSITIES: BASIC DEFINITION AND RESULTS}

It is well known and easy to prove that

$$
V_{V}(t, x)=\mathbb{P}\left(x \in \Theta^{t}\right) \quad \text { for } v^{d} \text {-a.e. } x \in \mathbb{R}^{d} .
$$

The so-called "causal cone" of a point $x$ at time $t$, denoted here by $\mathscr{C}(t, x)$, plays a fundamental role in evaluating $V_{V}(t, x)$. It is defined as the space-time region in which at least one nucleation event has to take place in order to cover the point $x$ at time $t$; namely, it is the subset of $\mathbb{R}_{+} \times \mathbb{R}^{d}$

$$
\mathscr{C}(t, x):=\left\{(s, y) \in[0, t] \times \mathbb{R}^{d}: x \in \Theta_{s}^{t}(y)\right\} .
$$

(see, e.g., also Rios and Villa, 2009, Sec 2.4.)

Let us observe that under our assumption above on $G$, it follows that

$$
\mathscr{C}(t, x)=\left\{\begin{array}{l}
B_{G t}(x) \\
\left\{(s, y) \in[0, t] \times \mathbb{R}^{d}: y \in B_{G(t-s)}(x)\right\}
\end{array}\right.
$$

in the site-saturated and in the time-dependent case, respectively. (For the general case of spacetime dependent growth rate, see, e.g., Villa (2008); Rios and Villa (2009) and references therein.) Quite general results on $V_{V}(t, x)$ in terms of the causal cone are proved in Villa (2008). In particular we recall here that

$$
V_{E}(t, x)=\Lambda(\mathscr{C}(t, x)),
$$


and that

$$
G=\frac{1}{S_{V}(t, x)} \frac{\partial V_{V}(t, x)}{\partial t}=\frac{1}{S_{E}(t, x)} \frac{\partial V_{E}(t, x)}{\partial t},
$$

moreover, under Poissonian assumption on the nucleation process it holds

$$
V_{V}(t, x)=1-\mathrm{e}^{-V_{E}(t, x)}
$$

and

$$
S_{V}(t, x)=\left(1-V_{V}(t, x)\right) S_{E}(t, x) .
$$

It is clear the importance of the relationships above in real applications. For instance, if the velocity $G$ is known, Eq. 2 can be used to find out the mean interfacial area density or the interfacial area per unit of volume from the corresponding mean volume density or volume fraction.

\section{GENERAL EXPRESSION IN THE CASE OF NUCLEATION ON LOWER DIMENSIONAL SETS}

In order to model the situation in which the nucleation takes place spatially in lower dimensional subsets and subspaces of $\mathbb{R}^{d}$, we shall make use of the so called "delta-formalism", as follows. Let $S_{n}$ be a $\mathscr{H}^{n}$-measurable subset of $\mathbb{R}^{d}$ with Hausdorff dimension $n \in\{1,2, \ldots, d-1\}$, having denoted by $\mathscr{H}^{n}$ the $n$-dimensional Hausdorff measure; then $\delta_{S_{n}}(y)$ is the so-called delta function associated to $S_{n}$ (which can be seen as a generalization to the well-known delta-function $\delta_{x_{0}}$ associated to a point $x_{0} \in \mathbb{R}^{d}$ ). It is the generalized function defined formally by

$$
\int_{A} \delta_{S_{n}}(y) \mathrm{d} y:=\mathscr{H}^{n}\left(S_{n} \cap A\right) \quad \forall A \in \mathscr{B}_{\mathbb{R}^{d}} .
$$

Thus, we may consider Poisson nucleation processes on $S_{n}$ with intensity measure $\Lambda$ given by

$$
\Lambda(\mathrm{d} y)=\lambda(y) \delta_{S_{n}}(y) \mathrm{d} y,
$$

and

$$
\Lambda(\mathrm{d}(s, y))=\lambda(s, y) \delta_{S_{n}}(y) \mathrm{d} s \mathrm{~d} y,
$$

in the site-saturated and in the time-dependent case, respectively.

Therefore $V_{V}(t, x)$ is given by Eq. 3 with

$$
V_{E}(t, x)=\left\{\begin{array}{l}
\int_{B_{G t}(x)} \lambda(y) \delta_{S_{n}}(y) \mathrm{d} y \\
\int_{0}^{t}\left(\int_{B_{G(t-s)}(x)} \lambda(s, y) \delta_{S_{n}}(y) \mathrm{d} y\right) \mathrm{d} s
\end{array}\right.
$$

in the site-saturated and in the time-dependent case, respectively.

Of course in real situations nucleation cannot take place on unbounded subsets; nevertheless there are situations in which it could be more convenient (because more tractable mathematically in order to obtain explicit formulas) to consider nucleation on unbounded domains, such as planes or lines, as we shall see in the sequel. Anyway, in order to model the real general case in which nucleation takes place in a bounded region $C$ (the sample where the reaction takes place) it is sufficient to consider the nucleation process having intensity measure $\Lambda$ of the type

$$
\Lambda(\mathrm{d} y)=\lambda(y) \delta_{S_{n} \cap C}(y) \mathrm{d} y,
$$

and

$$
\Lambda(\mathrm{d}(s, y))=\lambda(s, y) \delta_{S_{n} \cap C}(y) \mathrm{d} s \mathrm{~d} y,
$$

in the site-saturated and in the time-dependent case, respectively.

In what follows, we consider the case $d=3$ and $n=2$ or $n=1$ to model situations in which nucleation takes place on planes or lines, respectively, which are of particular interest in real applications. In particular, we shall consider the case in which the nucleation is homogenous in space in the sitesaturated case, and homogeneous in space and time in the time-dependent case, which corresponds to take $\lambda(y) \equiv \lambda>0$ and $\lambda(s, y) \equiv c>0$, respectively, in the above equations. This assumption allows us to provide explicit formulas for $V_{V}$ (and so for their related quantities), useful in various real applications. Indeed, under such homogeneity assumption the above expressions for $V_{E}$ simplify as follows:

$$
V_{E}(t, x)=\left\{\begin{array}{l}
\lambda \mathscr{H}^{n}\left(S_{n} \cap C \cap B_{G t}(x)\right), \\
c \int_{0}^{t} \mathscr{H}^{n}\left(S_{n} \cap C \cap B_{G(t-s)}(x)\right) \mathrm{d} s,
\end{array}\right.
$$

in the site-saturated and in the time-dependent case, respectively; so the problem reduces to evaluate the $\mathscr{H}^{n}$ measure of the intersection of a ball with the spatial region $\left(S_{n} \cap C\right)$ where the nucleation takes place. Note also that for point $x$ and time $t$ such that $B_{G t}(x) \subset C$ (for instance at the very beginning of a reaction or for points $x$ sufficiently far from the boundary of the sample), then $\mathscr{H}^{n}\left(S_{n} \cap C \cap\right.$ $\left.B_{G(t-s)}(x)\right)=\mathscr{H}^{n}\left(S_{n} \cap B_{G(t-s)}(x)\right)$ for any $s \in[0, t]$, so that the computation of $V_{E}$ might be simpler.

In the subsequent sections the constants $\lambda$ and $c$ in Eq. 4 will be denoted by $\lambda_{S}$ and $I_{S}$, respectively, in the case of nucleation on planes, and by $\lambda_{L}$ and $I_{L}$, respectively, in the case of nucleation on lines. Thus, $\lambda_{S}$ will represent the mean number of nuclei per unit of 
area of a plane, whereas $\lambda_{L}$ the mean number of nuclei per unit of length of a line, and $I_{S}$ and $I_{L}$ will represent the nucleation rate in the time-dependent nucleation (i.e., the mean number of nuclei per unit of time) in the plane case and the line case, respectively.

\section{NUCLEATION ON RANDOM PLANES}

In Villa and Rios (2010, supplementary material) explicit expressions for $V_{V}$ and $V_{E}$ in the case of nucleation on one fixed plane $B$ in $\mathbb{R}^{3}$ are provided, both in the case of time dependent nucleation and in the case of site-saturation. By denoting $r(x)$ the distance of a point $x \in \mathbb{R}^{3}$ to $B$, we recall that

$V_{E}(t, x)=\left\{\begin{array}{l}\lambda_{S} \pi\left(G^{2} t^{2}-r(x)^{2}\right) \mathbf{1}_{[0, G t]}(r(x)) \\ I_{S} \pi\left(\frac{2 r(x)^{3}}{3 G}-r(x)^{2} t+\frac{G^{2} t^{3}}{3}\right) \mathbf{1}_{[0, G t]}(r(x))\end{array}\right.$

in the site-saturated and in the time-dependent case, respectively, where $\mathbf{1}_{A}(a)$ is the indicator function of any set $A$.

Let us notice from the formulas above that the value of $V_{E}$ depends on the distance $r(x)$ from $x$ to the plane; thus, in order to find out explicit formulas for unions of random planes it is convenient to represent any plane $B$ by giving its orientation and distance from the origin. Indeed, we recall that a plane $B$ in $\mathbb{R}^{3}$ is uniquely determined by its distance from the origin, say $u$, and by its normal outer vector, say $w \in \mathbf{S}^{2}\left(\mathbf{S}^{2}\right.$ is the unit sphere in $\left.\mathbb{R}^{3}\right)$. The equation of $B=B(w, u)$ is then given by

$$
B(w, u):=\left\{x \in \mathbb{R}^{3}:\langle w, x\rangle=u\right\}
$$

and it is well known that $\operatorname{dist}(x, B)=|\langle w, x\rangle-u|$ for any $x \in \mathbb{R}^{3}(\langle w, x\rangle$ is the scalar product of $w$ and $x)$. As a consequence, by denoting $V_{V}^{u, w}(t, x)$ and $V_{E}^{u, w}(t, x)$ the corresponding mean volume density and the mean extended volume density, respectively, associated to the transformed region $\Theta^{t}=\Theta^{t}(w, u)$ at time $t$ due to the nucleation on the plane $B(w, u)$, it follows that

$$
V_{V}^{u, w}(t, x) \stackrel{\text { Eq. }}{=} 1-\mathbb{P}\left(x \notin \Theta^{t}(w, u)\right)=1-\mathrm{e}^{-V_{E}^{u, w}(t, x)},
$$

with

$$
\begin{aligned}
& V_{E}^{u, w}(t, x)= \\
& \left\{\begin{array}{l}
\lambda_{S} \pi\left(G^{2} t^{2}-|\langle w, x\rangle-u|^{2}\right) \mathbf{1}_{[0, G t]}(|\langle w, x\rangle-u|) \\
I_{S} \pi\left(\frac{2|\langle w, x\rangle-u|^{3}}{3 G}-|\langle w, x\rangle-u|^{2} t+\frac{G^{2} t^{3}}{3}\right) \\
\cdot \mathbf{1}_{[0, G t]}(|\langle w, x\rangle-u|)
\end{array}\right.
\end{aligned}
$$

in the site-saturated and in the time-dependent case, respectively.
Remark 1 In Cahn (1956, p. 451) a formula for the mean total volume at time $t$ (denoted by $V_{0}$ in that paper) occupied by the grains with nucleus on a unit area of $B$ is given in the site-saturated case. We may notice that such a formula coincide with the mean volume of $\Theta^{t}$ in the spatial region $A:=[0,1] \times[0,1] \times$ $[-\infty,+\infty]$, due to the time-dependent nucleation on the plane $B:=\left\{x_{3}=0\right\}$. Indeed, by Eqs. 1 and 6 we get

$$
\begin{array}{r}
\mathbb{E}\left[v^{3}\left(\Theta^{t} \cap A\right)\right] \\
=\int_{0}^{1} \int_{0}^{1}\left(\int_{-\infty}^{+\infty}\left(1-\mathrm{e}^{-I_{S} \pi\left(\frac{2\left|x_{3}\right|^{3}}{3 G}-\left|x_{3}\right|^{2} t+\frac{G^{2} t^{3}}{3}\right)}\right)\right. \\
\left.\cdot \mathbf{1}_{\left|x_{3}\right|<G t} \mathrm{~d} x_{3}\right) \mathrm{d} x_{1} \mathrm{~d} x_{2} \\
=2 \int_{0}^{G t}\left(1-\mathrm{e}^{-I_{S} \pi\left(\frac{2 x_{3}^{3}}{3 G}-x_{3}^{2} t+\frac{G^{2} t^{3}}{3}\right)}\right) \mathrm{d} x_{3},
\end{array}
$$

which coincides with the above mentioned result in Cahn's paper, after the change of variable $z=x_{3} / G t$.

\section{NUCLEATION ON ONE RANDOM PLANE}

Let us now consider the case in which $B$ is random, i.e let $B=B(W, D):=\left\{x \in \mathbb{R}^{3}:\langle W, x\rangle=D\right\}$ be a random plane in $\mathbb{R}^{3}$, where $W$ is a random unit vector in $\mathbf{S}^{2}$ with probability law $P_{W}$, and $D$ is the random distance from the origin, with probability law $P_{D}$. We denote by $P_{D, W}$ their joint probability on $\mathbb{R}_{+} \times \mathbf{S}^{2}$. It follows that the transformed region $\Theta^{t}$ at time $t$ is due to double stochasticity: the random location of the plane, and the random nucleation on the plane. Thus, observing that $P_{D, W}\left(\mathbb{R}_{+} \times \mathbf{S}^{2}\right)=1$, the following chain of equalities holds:

$$
\begin{aligned}
V_{V}(t, x) & =1-\mathbb{P}\left(x \notin \Theta^{t}\right) \\
& =1-\int_{\mathbb{R}_{+}} \int_{\mathbf{S}^{2}} \mathbb{P}\left(x \notin \Theta^{t}(w, u)\right) P_{D, W}(\mathrm{~d}(u, w)) \\
& =1-\int_{\mathbb{R}_{+}} \int_{\mathbf{S}^{2}} \exp \left\{-V_{E}^{u, w}(t, x)\right\} P_{D, W}(\mathrm{~d}(u, w)) \\
& =\int_{\mathbb{R}_{+}} \int_{\mathbf{S}^{2}}\left(1-\exp \left\{-V_{E}^{u, w}(t, x)\right\}\right) P_{D, W}(\mathrm{~d}(u, w)) \\
& =\int_{\mathbb{R}_{+}} \int_{\mathbf{S}^{2}} V_{V}^{u, w}(t, x) P_{D, W}(\mathrm{~d}(u, w))
\end{aligned}
$$

Analogously, we get that

$$
V_{E}(t, x)=\int_{\mathbb{R}_{+}} \int_{\mathbf{S}^{2}} V_{E}^{u, w}(t, x) P_{D, W}(\mathrm{~d}(u, w)) .
$$

By noticing that

$$
1-\mathrm{e}^{g(s) \mathbf{1}_{A}(s)}=\left(1-\mathrm{e}^{g(s)}\right) \mathbf{1}_{A}(s),
$$


then from Eqs. 5, 6 and 7 we obtain the following general expressions for $V_{V}$, for the site-saturated case:

$$
\begin{array}{r}
V_{V}(t, x)=\int_{\mathbb{R}_{+}} \int_{\mathbf{S}^{2}}\left(1-\exp \left\{-\lambda_{S} \pi\left(G^{2} t^{2}-|\langle w, x\rangle-u|^{2}\right)\right\}\right) \\
\cdot \mathbf{1}_{[0, G t]}(|\langle w, x\rangle-u|) P_{D, W}(\mathrm{~d}(u, w)), \quad \text { (8) }
\end{array}
$$

and for the time-dependent case:

$$
\begin{aligned}
V_{V}(t, x)= & \\
\int_{\mathbb{R}_{+}} \int_{\mathbf{S}^{2}}( & 1-\exp \left\{-I_{S} \pi\left(\frac{2|\langle w, x\rangle-u|^{3}}{3 G}-|\langle w, x\rangle-u|^{2} t\right.\right. \\
& \left.\left.\left.\quad+\frac{G^{2} t^{3}}{3}\right)\right\}\right) \mathbf{1}_{[0, G t]}(|\langle w, x\rangle-u|) P_{D, W}(\mathrm{~d}(u, w)) .
\end{aligned}
$$

\section{Example: uniform orientation and uniform distance from the origin}

As a simple example of applicability of the above formulas, let us consider the case in which $W$ is uniform in $\mathbf{S}^{2}$ and the distance $D$ from the origin is uniform in $[0, M]$, and they are independent. In such a case Eq. 8 becomes

$$
\begin{aligned}
& V_{V}(t, x)= \\
& \frac{1}{4 \pi M} \int_{0}^{M} \int_{\mathbf{S}^{2}}\left(1-\exp \left\{-\lambda_{S} \pi\left(G^{2} t^{2}-|\langle w, x\rangle-u|^{2}\right)\right\}\right) \\
& \mathbf{1}_{[0, G t]}(|\langle w, x\rangle-u|) \mathscr{H}^{2}(\mathrm{~d} w) \mathrm{d} u,
\end{aligned}
$$

by changing to spherical coordinates $\left(w_{1}=\right.$ $\sin \theta \cos \phi ; \quad w_{2}=\sin \theta \sin \phi ; \quad w_{3}=\cos \theta, \quad \theta \in$ $[0, \pi], \phi \in[0,2 \pi])$, it can be written in an even more explicit form, useful for practical purposes:

$$
\begin{gathered}
V_{V}(t, x)=\frac{1}{4 \pi M} \int_{0}^{2 \pi} \int_{0}^{\pi} \\
\left(\int _ { 0 } ^ { M } \left(1-\exp \left\{-\lambda_{S} \pi\left(G^{2} t^{2}-(f(\theta, \phi, x)-u)^{2}\right\}\right)\right.\right. \\
\left.\mathbf{1}_{[f(\theta, \phi, x)-G t, f(\theta, \phi, x)+G t]}(u) \mathrm{d} u\right) \sin \theta \mathrm{d} \theta \mathrm{d} \phi,
\end{gathered}
$$

where

$$
f(\theta, \phi, x):=x_{1} \sin \theta \cos \phi+x_{2} \sin \theta \sin \phi+x_{3} \cos \theta .
$$

Obviously, one might proceed similarly for the timedependent case.

\section{NUCLEATION ON UNION OF RANDOM PLANES}

In this section we model nucleation on union of random planes.

Assumptions: Let $B_{1}=B_{1}\left(W_{1}, D_{1}\right), \ldots, B_{M}=$ $\left.B_{M}\left(W_{M}, D_{M}\right)\right)$ independent random planes, distributed as $B=B(W, D)$, where $M$ is a positive integer-valued random variable with mean $\mathbb{E}[M]=m$; that is $m$ is the mean number of planes where nucleation takes place. We assume that $D_{1}, D_{2}, \ldots$ and $W_{1}, W_{2}, \ldots$ are independent and identically distributed as $D$ and $W$, respectively, and independent of $M$, and that $D$ is a continuous random variable (this implies that the probability that two or more planes $B_{i}$ coincide is zero).

Then, by denoting $\Theta_{B_{i}}^{t}$ the union of the grains with nuclei on $B_{i}$, the transformed region $\Theta^{t}$ at time $t$ is the random set given by

$$
\Theta^{t}=\bigcup_{i=1}^{M} \Theta_{B_{i}}^{t} .
$$

Theorem 2 Under the above Assumptions, we have that

$$
V_{E}(t, x)=m \int_{\mathbb{R}_{+}} \int_{\mathbf{S}^{2}} V_{E}^{u, w}(t, x) P_{D, W}(\mathrm{~d}(u, w)),
$$

and

$$
V_{V}(t, x)=1-\mathscr{G}_{M}\left(\int_{\mathbb{R}_{+}} \int_{\mathbf{S}^{2}} \mathrm{e}^{-V_{E}^{u, w}(t, x)} P_{D, W}(\mathrm{~d}(u, w))\right)
$$

where $\mathscr{G}_{M}$ is the probability generating function of $M$, and $V_{E}^{u, w}(t, x)$ is given by Eq. 6 .

Remark 3 Note that, if $t=0$, then $V_{V}(0, x)=1-$ $\mathscr{G}(1)=0$, as expected.

Proof. Given $M=n$, we know that $B_{1}, \ldots, B_{n}$ are independent with random distances $D_{i} \sim D$ and random orientation $W_{i} \sim W$. Given then the distances $D_{1}=u_{1}, \ldots, D_{n}=u_{n}$ of $B_{1}, \ldots, B_{n}$, respectively, to the origin, and given the orientations $W_{1}=w_{1}, \ldots, W_{n}=$ $w_{n}$, the mean volume extended density conditioned to $\left(n, u_{1}, \ldots, u_{n}, w_{1}, \ldots, w_{n}\right)$, say $V_{E}^{n, u_{1}, \ldots, u_{n}, w_{1}, \ldots, w_{n}}(t, x)$, is given by

$$
V_{E}^{n, u_{1}, \ldots, u_{n}, w_{1}, \ldots, w_{n}}(t, x)=\sum_{i=1}^{n} V_{E}^{u_{i}, w_{i}}(t, x)
$$

It follows that

$$
\begin{aligned}
V_{E}(t, x) & =\sum_{n} n \int_{\mathbb{R}_{+}} \int_{\mathbf{S}^{2}} V_{E}^{u, w}(t, x) P_{D, W}(\mathrm{~d}(u, w)) \mathbb{P}(M=n) \\
& =\int_{\mathbb{R}_{+}} \int_{\mathbf{S}^{2}} V_{E}^{u, w}(t, x) P_{D, W}(\mathrm{~d}(u, w)) \sum_{n} n \mathbb{P}(M=n) \\
& =m \int_{\mathbb{R}_{+}} \int_{\mathbf{S}^{2}} V_{E}^{u, w}(t, x) P_{D, W}(\mathrm{~d}(u, w)) .
\end{aligned}
$$


By noticing that

$$
\begin{aligned}
\underbrace{\int_{A} \cdots \int_{A}}_{n \text {-times }} f\left(a_{1}\right) f\left(a_{2}\right) \cdots f\left(a_{n}\right) \mathrm{d} a_{1} \cdots \mathrm{d} a_{n} & \\
= & {\left[\int_{A} f(a) \mathrm{d} a\right]^{n}, }
\end{aligned}
$$

we obtain

$$
\begin{aligned}
& V_{V}(t, x)=1-\mathbb{P}\left(x \notin \Theta^{t}\right) \\
& =1-\sum_{n} \underbrace{\int_{\mathbb{R}_{+}} \int_{\mathbf{S}^{2}} \cdots \int_{\mathbb{R}_{+}} \int_{\mathbf{S}^{2}}}_{n \text {-times }} \mathrm{e}^{-V_{E}^{n, u_{1}, \ldots, u_{n}, w_{1}, \ldots, w_{n}}(t, x)} \\
& \cdot P_{D, W}\left(\mathrm{~d}\left(u_{1}, w_{1}\right)\right) \cdots P_{D, W}\left(\mathrm{~d}\left(u_{n}, w_{n}\right)\right) \mathbb{P}(M=n) \\
& \stackrel{\text { Eq. } 11}{=} 1-\sum_{n} \underbrace{\int_{\mathbb{R}_{+}} \int_{\mathbf{S}^{2}} \ldots \int_{\mathbb{R}_{+}} \int_{\mathbf{S}^{2}} \prod_{i=0}^{n} \mathrm{e}^{-V_{E}^{u_{i}, w_{i}}(t, x)}}_{n \text {-times }} \\
& \cdot P_{D, W}\left(\mathrm{~d}\left(u_{1}, w_{1}\right)\right) \cdots P_{D, W}\left(\mathrm{~d}\left(u_{n}, w_{n}\right)\right) \mathbb{P}(M=n) \\
& =1- \\
& \sum_{n}\left(\int_{\mathbb{R}_{+}} \int_{\mathbf{S}^{2}} \mathrm{e}^{-V_{E}^{u, w}(t, x)} P_{D, W}(\mathrm{~d}(u, w))\right)^{n} \mathbb{P}(M=n) \\
& =1-\mathscr{G}(z),
\end{aligned}
$$

where $\mathscr{G}$ is the probability generating function of $M$, and

$$
z:=\int_{\mathbb{R}_{+}} \int_{\mathbf{S}^{2}} \mathrm{e}^{-V_{E}^{u, w}(t, x)} P_{D, W}(\mathrm{~d}(u, w))
$$

Remark 4 Let us notice that if $M$ is a Poisson random variable with mean $m$, then $\mathscr{G}_{M}(z)=\mathrm{e}^{-m(1-z)}$, and so, by Eq. 10 and $P_{D, W}\left(\mathbb{R}_{+} \times \mathbf{S}^{2}\right)=1$,

$$
\begin{aligned}
& V_{V}(t, x)= \\
& 1-\exp \left\{-m \int_{\mathbb{R}_{+}} \int_{\mathbf{S}^{2}}\left(1-\mathrm{e}^{-V_{E}^{u, w}(t, x)}\right) P_{D, W}(\mathrm{~d}(u, w))\right\}
\end{aligned}
$$

More numerically tractable expressions for the above equations can be obtained by passing into spherical coordinates as the example given in the previous section.

\section{PARTICULAR PROBABILITY DISTRIBUTIONS OF THE RANDOM ORIENTATION $W$ USEFUL IN APPLICATIONS}

Throughout this section, without any other specification, the number $M$ of planes is assumed to be a Poisson random variable, so that Eq. 12 holds. As a consequence, such a model can be seen as a network of Poisson hyperplanes in 3D (Matheron, 1975). We consider here a few particular cases of interest in applications.

\section{Parallel and orthogonal planes}

Let us consider the general case in which the distribution of the orientation $W$ is discrete, and assume that $D$ and $W$ are independent. Therefore $W$ is a discrete random vector in $\mathbf{S}^{2}$, that is there exist $w_{1}, w_{2}, \ldots$ in $\mathbf{S}^{2}$ and $p_{1}, p_{2}, \ldots$ in $[0,1]$ such that $\sum_{i} p_{i}=$ 1 and $\mathbb{P}\left(W=w_{i}\right)=p_{i}$ for all $i$. Then Eq. 12 simplifies as follows:

$$
\begin{aligned}
& V_{V}(t, x)= \\
& 1-\exp \left\{-m \int_{\mathbb{R}_{+}} \sum_{i}\left(\left(1-\mathrm{e}^{-V_{E}^{u, w_{i}}(t, x)}\right) p_{i}\right) P_{D}(\mathrm{~d} u)\right\}
\end{aligned}
$$

with $V_{E}^{u, w_{i}}(t, x)$ given by Eq. 6 .

A particular case of the above model is obtained by assuming that the planes are parallel. In such a case, the outer normal vector of each plane is fixed, say $\bar{w}=(0,0,1)$ (that is, the planes are parallel to the $x y$ plane); as a consequence we obtain

$$
V_{V}(t, x)=1-\exp \left\{-m \int_{\mathbb{R}_{+}}\left(1-\mathrm{e}^{-V_{E}^{u, \bar{w}}(t, x)}\right) P_{D}(\mathrm{~d} u)\right\} .
$$

An explicit expression in the case $D$ is uniformly distributed in $[0, \mathrm{~K}]$ is then given by

$$
\begin{aligned}
& V_{V}(t, x)= \\
& \left\{\begin{array}{c}
1-\exp \left\{-\frac{m}{K} \int_{0}^{K}\left(1-\mathrm{e}^{-\lambda_{S} \pi\left(G^{2} t^{2}-\left(x_{3}-u\right)^{2}\right)}\right)\right. \\
\left.\cdot \mathbf{1}_{\left[x_{3}-G t, x_{3}+G t\right]}(u) \mathrm{d} u\right\} \\
1-\exp \left\{-\frac{m}{K} \int_{0}^{K}\left(1-\mathrm{e}^{-I_{S} \pi\left(\frac{2\left|x_{3}-u\right|^{3}}{3 G}-\left|x_{3}-u\right|^{2} t+\frac{G^{2} t^{3}}{3}\right)}\right)\right. \\
\left.\cdot \mathbf{1}_{\left[x_{3}-G t, x_{3}+G t\right]}(u) \mathrm{d} u\right\}
\end{array}\right.
\end{aligned}
$$

in the site-saturated and in the time-dependent case, respectively.

Note that $m / K$ is the mean area fraction of the planes in $[0, K] \times[0,1]^{2}$.

Remark 5 Let us consider for instance the sitesaturated case. Since we assumed that each random plane $B_{i}$ is of the type $x_{3}=D$ with $D \sim U[0, K]$, and $\lambda_{S}$ nuclei per unit of area in mean for each plane, then nucleation takes place in the spatial region between the plane $x_{3}=0$ and the plane $x_{3}=K$ with $\lambda_{s} m$ nuclei in mean in $[0,1]^{2} \times[0, K]$. Thus, we expect that for 
$m$ sufficiently large, such a birth-and-growth process approximates one with nucleation process given by a homogeneous Poisson point process between the two parallel planes $x_{3}=0$ and $x_{3}=K$, with intensity $\lambda=$ $\lambda_{s} m / K$. Indeed, e.g., in the case $t>K / G$, for any $x=\left(x_{1}, x_{2}, x_{3}\right)$ with $x_{3} \in[0, K]$,

$$
\begin{array}{r}
V_{V}(t, x)=1-\exp \left(-\frac{m}{K} \int_{0}^{K}\left(1-\mathrm{e}^{-\lambda_{S} \pi\left(G^{2} t^{2}-\left(x_{3}-u\right)^{2}\right)}\right) \mathrm{d} u\right) \\
\sim 1-\exp \left\{1-\lambda \pi\left(G^{2} t^{2} K-\frac{K^{3}}{3}+K^{2} x_{3}-M x_{3}^{2}\right)\right\},
\end{array}
$$

that coincides with Eq.(101) in Villa and Rios (2010), where the case of nucleation between two parallel planes is studied.

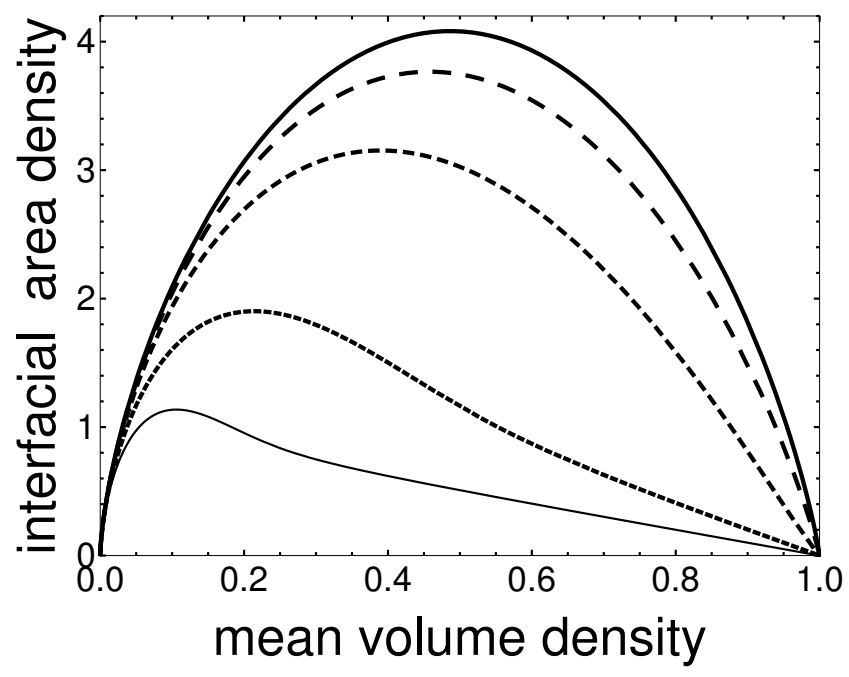

Fig. 1. Microstructural path for transformations nucleated on random points and planes. The solid black line: homogeneous Poisson point process with intensity $\lambda=10$. The other lines represent a transformation nucleated on $m$ planes $x_{3}=[-K, K]$ with $\lambda_{S}$ number of nuclei per unit of area, so that $\lambda_{S} m / 2 K=10$ : a)thin solid line $\lambda_{S}=$ 20; b) short-dashed line: $\lambda_{S}=10 ;$ c)medium-dashed line: $\left.\lambda_{S}=\sqrt{10} ; d\right)$ dashed line: $\lambda_{S}=1$. The clustering effect is accentuated for low $m / 2 K$ and high $\lambda_{S}$, thin solid line. In contrast for high $m / 2 K$ and low $\lambda_{S}$, dashed line, the microstructural path approaches that of a reaction nucleated according with a homogeneous Poisson point process, solid curve.

Fig. 1 illustrates the above Remark. The thick solid line is the microstructural path, evaluated in $x=0$, of a transformation for which nuclei are located in space according to a homogeneous Poisson point process with intensity $\lambda=10$. The other lines represent transformations nucleated on $m$ planes $x_{3} \in[-K, K]$, with $\lambda_{S}$ number of nuclei per unit of area, so that $\lambda_{S} m / 2 K=10$ The thin solid line represents the case in which $m / 2 K=0.5$ and $\lambda_{S}=20$. A pronounced lefthand skew is apparent. As the number of nuclei per unit of area decreases and the area per unit of volume increases (thus keeping the total number of nuclei per unit of volume constant) the curves progressively approach the thick solid line as expected. Preferential nucleation on planes and lines may be understood as a clustering effect according to Vandermeer (2005). It is the severity of this clustering that shows as an asymmetry in the microstructural path plot. Such a clustering effect is more intense for low $m / 2 K$ and high $\lambda_{s}$.

It might be of interest also to model nucleations on orthogonal planes. Since in many real applications it is of interest the behavior of the process in the centre of the specimen, for sake of simplicity we shall assume here that the distance $D$ from the origin is uniformly distributed in $[0, K]$, so that it is easy to get an explicit expression for $V_{V}(t, x)$, which might be used to represent the mean volume density at points $x$ sufficiently far from to the boundary of the specimen. Therefore, denoted by $w_{1}=(1,0,0), w_{2}=(0,1,0)$, and $w_{3}=(0,0,1)$, with $\mathbb{P}\left(W=w_{i}\right)=p_{i}$, we obtain

$$
\begin{aligned}
& V_{V}(t, x) \stackrel{\text { Eqs. }}{=} 13,5 \\
& \left\{\begin{array}{r}
1-\exp \left\{-\frac{m}{K} \int_{0}^{K} \sum_{i=1}^{3} p_{i}\left(1-\mathrm{e}^{-\lambda_{S} \pi\left(G^{2} t^{2}-\left(x_{i}-u\right)^{2}\right)}\right)\right. \\
\left.\mathbf{1}_{\left[x_{i}-G t, x_{i}+G t\right]}(u) \mathrm{d} u\right\} \\
1-\exp \left\{-\frac{m}{M} \int_{0}^{M} \sum_{i=1}^{3} p_{i}\left(1-\mathrm{e}^{\frac{2\left|x_{i}-u\right|^{3}}{3 G}-\left(x_{i}-u\right)^{2} t+\frac{G^{2} t^{3}}{3}}\right)\right. \\
\left.\mathbf{1}_{\left[x_{i}-G t, x_{i}+G t\right]}(u) \mathrm{d} u\right\}
\end{array}\right.
\end{aligned}
$$

in the site-saturated and in the time-dependent case, respectively.

\section{An example of continuous not-uniform distribution of $W$}

Let us consider the following simple model where nucleation takes place on planes having continuous but not uniform orientation. It may be used as model for recrystallization processes in which nucleation takes places on ellipsoidal grains, for instance.

For sake of simplicity we consider here the sitesaturated case, and we assume, as above, that the distance $D$ from the origin in uniformly distributed, but now dependent on the orientation of the plane. Thus, denoted by $P_{D \mid W}(\mathrm{~d} u \mid w)$ the conditioned probability law of $D$ given $W$, let us assume 


$$
\begin{aligned}
& P_{D \mid W}(\mathrm{~d} u \mid w):= \\
& \quad \frac{1}{M\left(\alpha w_{1}^{2}+\beta w_{2}^{2}+\gamma w_{3}^{2}\right)} \mathbf{1}_{\left[0, M\left(\alpha w_{1}^{2}+\beta w_{2}^{2}+\gamma w_{3}^{2}\right)\right]}(u) \mathrm{d} u,
\end{aligned}
$$

and

$P_{W}(\mathrm{~d} w):=\frac{3}{4 \pi} \frac{a^{2} w_{1}^{2}+b^{2} w_{2}^{2}+c^{2} w_{3}^{2}}{a^{2}+b^{2}+c^{2}} \mathbf{1}_{\mathbf{S}^{2}}(w) \mathscr{H}^{2}(\mathrm{~d} w)$,

where $a:=1 / \sqrt{\alpha}, b:=1 / \sqrt{\beta}$, and $c:=1 / \sqrt{\gamma}$.

Then it follows

$$
\begin{aligned}
& V_{V}(t, x)=1-\exp \left\{-\frac{3 m}{4 \pi\left(a^{2}+b^{2}+c^{2}\right)}\right. \\
& \int_{0}^{2 \pi} \int_{0}^{\pi} \eta(u ; \theta, \phi)\left(a^{2} \sin ^{2} \theta \cos ^{2} \phi\right. \\
& \left.\left.+b^{2} \sin ^{2} \theta \sin ^{2} \phi+c^{2} \cos ^{2} \theta\right) \sin \theta \mathrm{d} \theta \mathrm{d} \phi\right\}
\end{aligned}
$$

with

$$
\begin{gathered}
\eta(u ; \theta, \phi):=\int_{0}^{M\left(\alpha \sin ^{2} \theta \cos ^{2} \phi+\beta \sin ^{2} \theta \sin ^{2} \phi+\gamma \cos ^{2} \theta\right)} \\
\frac{\left(1-\exp \left\{-\lambda_{S} \pi\left(G^{2} t^{2}-(f(\theta, \phi, x)-u)^{2}\right)\right\}\right)}{M\left(\alpha \sin ^{2} \theta \cos ^{2} \phi+\beta \sin ^{2} \theta \sin ^{2} \phi+\gamma \cos ^{2} \theta\right)} \\
\cdot \mathbf{1}_{[f(\theta, \phi, x)-G t, f(\theta, \phi, x)+G t]}(u) \mathrm{d} u
\end{gathered}
$$

and $f(\theta, \phi, x)$ given by Eq. 9 .

Remark 6 Note that the case of uniform distribution of $W$ in $\mathbf{S}^{2}$, with $W$ and $D$ independent, follows as a particular case with $a=b=c=1$.

\section{NUCLEATION ON RANDOM LINES}

As mentioned before, nucleation on lines might be used as model for nucleation on the edges of grains in recrystallization processes. Thus, let $L$ be a fixed line in $\mathbb{R}^{3}$, and $\lambda_{L}$ and $I_{L}$ be as in Sec "General expression in the case of nucleation on lower dimensional sets". Then it is not difficult to see that Eq. 4 becomes in this case

$$
\begin{aligned}
& V_{E}(t, x)= \\
& \left\{\begin{array}{l}
\lambda_{L} 2 \sqrt{G^{2} t^{2}-r(x)^{2}} \mathbf{1}_{[0, G t]}(r(x)) \\
I_{L} G\left[t \sqrt{t^{2}-(r(x) / G)^{2}}-(r(x) / G)^{2}\right. \\
\left.\cdot \log \left(\frac{t+\sqrt{t^{2}-(r(x) / G)^{2}}}{r(x) / G}\right)\right] \mathbf{1}_{[0, G t]}(r(x))
\end{array}\right.
\end{aligned}
$$

in the site-saturated and in the time-dependent case, respectively, where $r(x)$ is the distance of $x \in \mathbb{R}^{3}$ from $L$.

By following the representation given in Stoyan et al. (1995, Sec. 8.5)) (see also Matheron (1975)), we may uniquely determine a line $L$ in $\mathbb{R}^{3}$ with positive distance $u$ from the origin by $u$ and by a pair $(v, w) \in T\left(\mathbf{S}^{2}\right)$, being $T\left(\mathbf{S}^{2}\right)$ the tangent bundle to $\mathbf{S}^{2}$, that is the collection of pairs $(v, w)$ with $v \in \mathbf{S}^{2}$ and $w$ unit vector tangent to $\mathbf{S}^{2}$ at $v$. In other words, $v$ is the outer normal vector to the plane on which the line lies, while $w$ is the direction of the line. If $u=0$, only the direction $w$ is needed to identify the line, so $T\left(\mathbf{S}^{2}\right)$ has to be replaced by $\mathbf{S}^{2}$ and $L$ is the line $L(0, w)$ through the origin with direction $w \in \mathbf{S}^{2}$. We are going to consider nucleation on random lines of interest for real applications, whose distance from the origin is random with continuous distribution, so that $\mathbb{P}(u=0)=0$. Then, let us denote by $L^{u,(v, w)}$ the line having distance $u$ from the origin uniquely determined by the pair $(v, w) \in T\left(\mathbf{S}^{2}\right)$; it follows that $L^{u,(v, w)}$ is the line through the point $u v$ and direction $w$, and so

$$
\begin{array}{r}
\operatorname{dist}\left(x, L^{u,(v, w)}\right)= \\
\sqrt{\left(x_{1}-u v_{1}\right)^{2}+\left(x_{2}-u v_{2}\right)^{2}+\left(x_{3}-u v_{3}\right)^{2}-\langle x-u v, w\rangle^{2}} \\
:=j(x ; u,(v, w))
\end{array}
$$

As a consequence, denoted by $V_{V}^{u,(v, w)}(t, x)$ and $V_{E}^{u,(v, w)}(t, x)$ the corresponding mean volume density and the mean extended volume density, respectively, associated to the transformed region $\Theta^{t}=\Theta^{t}(u,(v, w))$ at time $t$ due to the Poissonian nucleation on the line $L^{u,(v, w)}$, it follows that

$$
\begin{aligned}
V_{V}^{u,(v, w)}(t, x) & =1-\mathbb{P}\left(x \notin \Theta^{t}(u,(v, w))\right) \\
& =1-\exp \left(-V_{E}^{u,(v, w)}(t, x)\right),
\end{aligned}
$$

with

$$
\begin{aligned}
& V_{E}^{u,(v, w)}(t, x)= \\
& \left\{\begin{array}{c}
2 \lambda_{L} \sqrt{G^{2} t^{2}-j^{2}(x ; u,(v, w))} \mathbf{1}_{[0, G t]}(j(x ; u,(v, w))) \\
I_{L} G\left[t \sqrt{t^{2}-(j(x ; u,(v, w)) / G)^{2}}-(j(x ; u,(v, w)) / G)^{2}\right. \\
\left.\cdot \log \left(\frac{t+\sqrt{t^{2}-(j(x ; u,(v, w)) / G)^{2}}}{j(x ; u, v, w)) / G}\right)\right] \mathbf{1}_{[0, G t]}(j(x ; u,(v, w)))
\end{array}\right.
\end{aligned}
$$

in the site-saturated and in the time-dependent case, respectively.

By proceeding along the same lines of the previous sections in the case of nucleation on a random line $L^{D,(V, W)}$ with $D$ and $(V, W)$ random quantities 
with probability law $P_{D}$ on $\mathbb{R}_{+}$, and $P_{T}$ on $T\left(\mathbf{S}^{2}\right)$, respectively, we have that

$$
\begin{array}{r}
V_{V}(t, x)=\int_{\mathbb{R}_{+}} \int_{T\left(\mathbf{S}^{2}\right)}\left(1-\exp \left\{-V_{E}^{u,(v, w)}(t, x)\right\}\right) \\
\cdot P_{D, T}(\mathrm{~d}(u,(v, w)),
\end{array}
$$

and

$$
V_{E}(t, x)=\int_{\mathbb{R}_{+}} \int_{T\left(\mathbf{S}^{2}\right)} V_{E}^{u,(v, w)}(t, x) P_{D, T}(\mathrm{~d}(u,(v, w)),
$$

having denoted by $P_{D, T}$ the joint probability of $D$ and $(V, W)$ on $\mathbb{R}_{+} \times T\left(\mathbf{S}^{2}\right)$. Whereas, by considering now the nucleation on unions of $M$ independent random lines $L_{1}, \ldots, L_{M}$ as $L^{D,(V, W)}$, with $\mathbb{E}[M]=m$, and denoted by $\Theta_{L_{i}}^{t}$ the union of the grains with nuclei on $L_{i}$, the transformed region $\Theta^{t}$ at time $t$ is the random set given by $\Theta^{t}=\bigcup_{i=1}^{M} \Theta_{L_{i}}^{t}$. Then, by proceeding similarly to the case of random planes, we obtain

$$
\begin{aligned}
& V_{V}(t, x)= \\
& 1-\mathscr{G}_{M}\left(\int_{\mathbb{R}_{+}} \int_{T\left(\mathbf{S}^{2}\right)} \mathrm{e}^{-V_{E}^{u(v, w)}(t, x)} P_{D, T}(\mathrm{~d}(u,(v, w))),\right.
\end{aligned}
$$

and if $M$ has Poisson distribution

$$
\begin{aligned}
V_{V}(t, x)=1- & \exp \left\{-m \int_{\mathbb{R}_{+}} \int_{T\left(\mathbf{S}^{2}\right)}\right. \\
& \left(1-\mathrm{e}^{-V_{E}^{u,(v, w)}(t, x)}\right) P_{D, T}(\mathrm{~d}(u,(v, w))\} .
\end{aligned}
$$

The model proposed above might be particularly useful in real applications when the behavior at the origin would be investigated; indeed, by noticing that $j(0 ; u,(v, w))=u$, it follows that $V_{V}(t, 0)$ does not depend on the orientation of the lines, but only on their distance $D$ from the origin.

Nevertheless, in dependence of different purposes it might be useful to consider different line models; for instance, by defining the random lines by a point process of their intersection with planes containing the origin and with different orientations, or, more simply, if the mean number of lines is finite (as occurs in applications), by giving a finite (in mean) collection $\left\{A_{i}, W_{i}\right\}$ of random points $A_{i}$ in $\mathbb{R}^{3}$ and random directions $W_{i}$ in $\mathbf{S}^{2}$, so that $L\left(A_{i}, W_{i}\right):=\left\{A_{i}+t W_{i}: t \in\right.$ $\mathbb{R}\}$ is the random line through $A_{i}$ with direction $W_{i}$. By observing that for any $x, a \in \mathbb{R}^{3}$ and $w \in \mathbf{S}^{2}$

$$
\begin{aligned}
& \operatorname{dist}(x, L(a, w))= \\
& \sqrt{\left(x_{1}-a_{1}\right)^{2}+\left(x_{2}-a_{2}\right)^{2}+\left(x_{3}-a_{3}\right)^{2}-\langle x-a, w\rangle^{2}} \\
& :=h(x ; a, w),
\end{aligned}
$$

and denoted by $V_{V}^{a, w}(t, x)$ and $V_{E}^{a, w}(t, x)$ the mean volume density and the mean extended volume density, respectively, associated to the transformed region at time $t$ due to the nucleation on the line $L(a, w)$, it follows that

$$
V_{V}^{a, w}(t, x)=1-\mathrm{e}^{-V_{E}^{a, w}(t, x)}
$$

with

$$
\begin{aligned}
& V_{E}^{a, w}(t, x) \stackrel{\text { Eq. } 14}{=} \\
& \left\{\begin{array}{c}
\lambda_{L} 2 \sqrt{G^{2} t^{2}-h^{2}(x ; a, w)} \mathbf{1}_{[0, G t]}(h(x ; a, w)) \\
I_{L} G\left[t \sqrt{t^{2}-(h(x ; a, w) / G)^{2}}-(h(x ; a, w) / G)^{2}\right. \\
\left.\log \left(\frac{t+\sqrt{t^{2}-(h(x ; a, w) / G)^{2}}}{h(x ; a, w) / G}\right)\right] \mathbf{1}_{[0, G t]}(h(x ; a, w))
\end{array}\right.
\end{aligned}
$$

in the site-saturated and in the time-dependent case, respectively. Therefore, similarly as above, we may consider now the nucleation on the union of random lines $L\left(A_{1}, W_{1}\right), \ldots, L\left(A_{M}, W_{M}\right)$, with $\mathbb{E}[M]=m \in \mathbb{R}$ and $\left\{\left(A_{i}, W_{i}\right)\right\}$ independent and identically distributed as $(A, W)$ with joint probability distribution $P_{A, W}$ on $\mathbb{R}^{3} \times \mathbf{S}^{2}$ and independent of $M$. Under the assumption that $A$ is a continuous random point (so, the probability that two or more lines $L_{i}$ coincide is zero), we get

$$
V_{E}(t, x)=m \int_{\mathbb{R}^{3}} \int_{\mathbf{S}^{2}} V_{E}^{a, w}(t, x) P_{A, W}(\mathrm{~d}(a, w)),
$$

and

$$
V_{V}(t, x)=1-\mathscr{G}_{M}\left(\int_{\mathbb{R}^{3}} \int_{\mathbf{S}^{2}} \mathrm{e}^{-V_{E}^{a, w}(t, x)} P_{A, W}(\mathrm{~d}(a, w))\right) .
$$

Such a model makes the particular case of nucleation on parallel lines easier to study, with respect to the previous one, as shown in the next section.

\section{PARALLEL LINES}

A particular case of the above model is obtained assuming that the lines are parallel. In such a case, the direction of each line is fixed, say $\bar{w}=(0,0,1)$ (that is the lines are parallel to the $z$-axis); as a consequence, by assuming $M \sim P o(m)$, Eq. 17 simplifies as follows

$$
V_{V}(t, x)=1-\exp \left\{-m \int_{\mathbb{R}^{3}}\left(1-\mathrm{e}^{-V_{E}^{a, \bar{w}}(t, x)}\right) P_{A}(\mathrm{~d} a)\right\}
$$

with $V_{E}^{a, \bar{w}}(t, x)$ as in Eq. 16 and noticing that $h(x ; a, \bar{w})=\sqrt{\left(x_{1}-a_{1}\right)^{2}+\left(x_{2}-a_{2}\right)^{2}}$.

Remark 7 Let us consider for instance the sitesaturated case. If in particular $A$ is uniformly 
distributed in $[0, K]^{3}$, then

$$
\begin{aligned}
& V_{V}(t, x)=1- \\
& \exp \left\{-\frac{m}{K^{2}} \int_{[0, K]^{2}}\left(1-\mathrm{e}^{-\lambda_{L} 2 \sqrt{\left.G^{2} t^{2}-\left(\left(x_{1}-a_{1}\right)^{2}+\left(x_{2}-a_{2}\right)^{2}\right)\right)}}\right)\right. \\
& \left.\mathbf{1}_{[0, G t]}\left(\sqrt{\left(x_{1}-a_{1}\right)^{2}+\left(x_{2}-a_{2}\right)^{2}}\right) \mathrm{d} a_{1} \mathrm{~d} a_{2}\right\}
\end{aligned}
$$

Note that $\frac{m}{K^{2}}$ is the length volume fraction of the lines in $[0, K]^{3}$, and that the mean number of nuclei on the lines in $[0, K]^{3}$ is $\lambda_{L} m K$.

Similarly to the case of nucleation on random parallel planes discussed in Remark 5, if the mean number $m$ of lines is sufficiently large, we expect that for any point $x$ not too close to the boundary of $[0, K]^{2} \times(-\infty,+\infty)$ (in particular for any $x$ such that $G t \leq x_{1} \leq K-G t$ and $\left.G t \leq x_{2} \leq K-G t\right)$, the value of $V_{V}(t, x)$ have to approximate that one associated to a Poisson homogeneous nucleation with intensity $\lambda=\lambda_{L} \frac{m}{K^{2}}$.

Indeed, for any $x$ such that $G t \leq x_{i} \leq K-G t$ for $i=1,2$,

$$
\begin{aligned}
& V_{V}(t, x) \\
& \stackrel{\text { Eq. } 18}{=} 1-\exp \left\{-\frac{m}{K^{2}} \int_{0}^{G t} \int_{0}^{2 \pi}\left[1-\mathrm{e}^{-2 \frac{\lambda}{\frac{m}{K^{2}}} \sqrt{G^{2} t^{2}-\rho^{2}}}\right]\right. \\
& \cdot \rho \mathrm{d} \theta \mathrm{d} \rho\} \\
& \quad \sim 1-\exp \left\{-4 \pi \lambda \int_{0}^{G t} \sqrt{G^{2} t^{2}-\rho^{2}} \rho \mathrm{d} \rho\right\} \\
& =1-\mathrm{e}^{-\frac{4}{3} \pi \lambda G^{3} t^{3}},
\end{aligned}
$$

which is just the mean volume density associated to a homogeneous Poisson nucleation with intensity $\lambda$.

\section{SUMMARY AND CONCLUSIONS}

- General expressions were derived for the mean volume density of the transformed phase when nucleation takes place on random planes and lines in $\mathbb{R}^{3}$. The general expression makes no assumption on the distribution of planes and of lines in space.

- Using superposition of processes (see, e.g., Stoyan et al., 1995), one may derive an expression for the situation in which nucleation takes place simultaneously on planes (grain boundaries) and lines (grain edges) and points (grain corners). Namely, we recall that if $\Phi_{1}, \ldots, \Phi_{n}$ are independent nucleation processes, then

$$
V_{V}(t, x)=1-\prod_{i=1}^{n}\left(1-V_{V}^{i}(t, x)\right),
$$

having denoted by $V_{V}^{i}$ the mean volume density associated with the nucleation process $\Phi_{i}$. Note that nucleation on grain corners may be easily modelled by point processes. Thus, by assuming simultaneous independent nucleation on planes, lines and points, and by denoting $V_{V}^{S}, V_{V}^{L}$ and $V_{V}^{P}$ the corresponding mean volume densities, we have

$$
\begin{array}{rl}
V_{V}(t, x) \stackrel{\text { Eq. }}{=} 19 & 1-\left(1-V_{V}^{S}(t, x)\right) \\
& \cdot\left(1-V_{V}^{L}(t, x)\right)\left(1-V_{V}^{P}(t, x)\right) .
\end{array}
$$

We point out that, even if the independence assumption may seem to be too restrictive for practical purposes, actually it is often implicitly assumed in modelling various situations. For instance, analysis of experimental results with the assumption of independence are discussed in Rios and Padilha (2003); Vandermeer and Rath (1989a;b).

- This paper provides the mathematical basis for the development of more specific expressions to be used in practical applications. By studying particular cases of practical relevance new expressions, such as the expression for parallel planes, may be obtained. These expressions significantly increase the scope of exactly solvable cases available to formal kinetics.

\section{ACKNOWLEDGEMENTS}

P.R.R. is grateful to the Conselho Nacional de Desenvolvimento Científico e Tecnológico, CNPq, and to the Fundação de Amparo à Pesquisa do Estado do Rio de Janeiro, FAPERJ, for financial support. P.R.R. would like to thank the Alexander von Humboldt Foundation for the Humboldt Research Award and Professor Günter Gottstein for his hospitality at the Institut für Metallkunde und Metallphysik, RWTHAachen, where this work was carried out. E.V. would also like to thank Professor Günter Gottstein for the financial support during her stay in Aachen. The authors would also like to thank the anonymous reviewers for useful comments and suggestions that significantly improved the paper.

\section{REFERENCES}

Aquilano D, Capasso V, Micheletti A, Patti S, Pizzocchero L, Rubbo M (2009). A birth and growth model for kinetic-driven crystallization processes. I. Modeling. Nonlinear Anal Real 10:71-92.

Avrami MJ (1939). Kinetics of phase change. I. General theory. J Chem Phys 7:1103-12. 
Avrami MJ (1940). Kinetics of phase change. II. Transformation-time relations for random distribution of nuclei. J Chem Phys 8:212-24.

Avrami MJ (1941). Granulation phase change, and microstructure kinetics of phase change. III. J Chem Phys 9:177-84.

Burger M, Capasso V, Micheletti C, Salani C (2002a). Mathematical models for polymer crystallization processes. In: Capasso V, ed. Mathematical modelling for polymer industry. Math in Industry Series, vol. 2 (2002) Berlin, Springer Verlag, 167-242.

Burger M, Capasso V, Salani C (2002b). Modelling multidimensional crystallization of polymers in interaction with heat transfer. Nonlinear Anal Real 3:139-60.

Cahn JW (1956). The kinetics of grain boundary nucleated reactions. Acta Metall 4:449-59.

Cahn JW (1996). The time cone method for nucleation and growth kinetics on a finite domain. Mat Res Soc Proc 398:425-37.

Capasso V, Villa E (2007a). On the evolution equation of mean geometric densities for a class of space and time inhomogeneous stochastic birth-and-growth processes. In: Weil W, ed. Stochastic Geometry, Berlin: Springer, 267-81.

Capasso V, Villa E (2007b). On mean densities of inhomogeneous geometric processes arising in material science and medicine. Image Anal Stereol 26:23-36.

Fanfoni M, Tomellini M (2005). Film growth viewed as stochastic dot processes. J Phys Condens Mat 17:R571.

Armstrong RA, Jackson JL, Glass L (1974). Dynamics of expanding inhibitory fields. Science 183:444-6.

Johnson WA, Mehl RF (1939). Reaction kinetics in process of nucleation and growth. Trans AIME 135:416-42.

Jun S, Bechhoefer J (2005). Nucleation and growth in one dimension. II. Application to DNA replication kinetics. Phys Rev E 71:011909.

Kolmogorov AN (1937). On the statistical theory of metal crystallization. Izv Akad Nauk USSR Ser Mat 1:355-9.

Kondo T, Sakuma T, Rios PR (1998). Application of microstructural path analysis to abnormal grain growth of $\mathrm{BaTiO}_{3}$ with an excess $\mathrm{TiO}_{2}$. Scr Mater 39:1713-7.

Matheron G (1975). Random sets and integral geometry. New York: Wiley.
Ramos RA, Rikvold PA, Novotny MA (1999). Test of the Kolmogorov-Johnson-Mehl-Avrami picture of metastable decay in a model with microscopic dynamics. Phys Rev B 59:9053-69.

Rios PR, Guimaraes JRC (2007). Microstructural path analysis of athermal martensite. Scr Mater 57:1105-8.

Rios PR, Guimaraes JRC (2008). Formal analysis of isothermal martensite spread. Mat Res 11:103-8.

Rios PR, Padilha AF (2003). Microstructural path of recrystallization in a commercial Al-Mn-Fe-Si (AA3003) Alloy. Mat Res 6:605-13.

Rios PR, Villa E (2009). Transformation kinetics for inhomogeneous nucleation. Acta Mater 57:1199-208.

Rios PR, Yamamoto T, Kondo T, Sakuma T (1998). Abnormal grain growth kinetics of $\mathrm{BaTiO}_{3}$ with an excess $\mathrm{TiO}_{2}$. Acta Mater 46:1617-23.

Starink MJ (2004). Analysis of aluminium based alloys by calorimetry: quantitative analysis of reactions and reaction kinetics. Int Mater Rev 49:191-226.

Stoyan D, Kendall WS, Mecke J (1995). Stochastic Geometry and its Application. Chichester: Wiley.

Tomellini M, Fanfoni M (2008). Impingement factor in the case of phase transformations governed by spatially correlated nucleation. Phys Rev B 78:014206.

Vandermeer RA (2005). Microstructural descriptors and the effects of nuclei clustering on recrystallization path kinetics. Acta Mater 53:1449-57.

Vandermeer RA, Jensen DJ (2001). Microstructural path and temperature dependence of recrystallization in commercial aluminum. Acta Mater 49:2083-94.

Vandermeer RA, Rath BB(1989a). Modeling recrystallization kinetics in a deformed iron single crystal. Metall Trans A 20:391-401.

Vandermeer RA, Rath BB(1989b). Microstructural modeling of recrystallization in deformed iron single crystals. Metall Trans A 20:1933-42.

Villa E (2008). A note on mean volume and surface densities for a class of birth-and-growth stochastic processes. Int J Contemp Math Sci 3:1141-55.

Villa E, Rios PR (2009). Transformation kinetics for nuclei clusters. Acta Mater 57:3714-24.

Villa E, Rios PR (2010). Transformation kinetics for surface and bulk nucleation. Acta Mater 58:2752-68. 